\title{
Endothelial Progenitor Cells Contribute to the Vascularization of Endometriotic Lesions
}

Matthias W. Laschke, Christian Giebels, Ruth M. Nickels, Claudia Scheuer, and Michael D. Menger

From the Institute for Clinical and Experimental Surgery, University of Saarland, Homburg/Saar, Germany

Endometriosis is a frequent gynecological disease that is characterized by the development of vascularized endometriotic lesions inside the peritoneal cavity. Herein, we analyzed whether circulating endothelial progenitor cells (EPCs) are recruited and incorporated into the microvasculature of these lesions. Intraperitoneal endometriotic lesions were surgically induced in irradiated FVB/N mice, which were reconstituted with bone marrow from $\mathrm{FVB} / \mathrm{N}-\mathrm{TgN}$ (Tie2/green fluorescent protein [GFP]) 287 Sato mice. Vascularization and recruitment of GFP-positive EPCs in the lesions was analyzed by intravital fluorescence microscopy and immunohistochemistry over 4 weeks. The numbers of stem cell antigen-1 (Sca-1)/vascular endothelial growth factor receptor-2positive EPCs in blood and hematopoietic organs of additional endometriotic and control mice were assessed by flow cytometry. We found that approximately $15 \%$ of the microvascular endothelium in engrafting endometriotic lesions consisted of incorporated GFPpositive EPCs. Recruitment of EPCs into the lesions coincided with the establishment of own blood supply and the expression of stromal cell-derived factor-1. Accordingly, treatment with the stromal cell-derived factor-1/chemokine receptor type 4 axis antagonist AMD3100 significantly decreased the number of recruited EPCs and the vascularization of endometriotic lesions. However, endometriosis did not induce increased levels of EPCs in the blood, bone marrow, and spleen of $\mathrm{C} 57 \mathrm{BL} / 6$ mice. To our knowledge, our findings indicate for the first time that vasculogenesis (ie, de novo generation of blood vessels from EPCs) may represent an integral mechanism in the pathogenesis of endometriosis. (Am J Pathol 2011, 178:442-450; DOI: 10.1016/j.ajpath.2010.11.037)

Endometriosis is one of the most frequent gynecological diseases and is defined as the presence and proliferation of endometrial tissue outside the uterine cavity, also referred to as endometriotic lesions. Although the pathogenesis of endometriosis is still a matter of discussion, it is widely believed that these lesions originate from shed endometrial fragments that enter the peritoneal cavity through the fallopian tubes by retrograde menstruation. ${ }^{1}$ Because they initially lack their own blood supply, rapid vascularization is a major requirement for their long-term survival and growth at ectopic sites. ${ }^{2}$ Accordingly, endometriosis represents a typical angiogenic disease that is characterized by hypoxia-induced up-regulation of proangiogenic growth factors, formation of new microvascular networks, and adaptive vascular remodeling processes dependent on tissue perfusion. ${ }^{3}$

During the past years, several animal and human studies $^{4,5}$ have indicated that circulating endothelial progenitor cells (EPCs) from the bone marrow contribute to various postnatal angiogenic processes. For instance, EPCs have been involved in neovascularization during atherosclerosis, ${ }^{6}$ remodeling after myocardial infarction, ${ }^{7}$ fracture healing, ${ }^{8}$ retinal angiogenesis, ${ }^{9}$ and tumor growth. ${ }^{10}$ Typically, EPCs are recruited into areas of endothelial injury and ischemia, a process that is regulated by the close interaction of soluble or surface-arrested angiogenic CC- and CXC-chemokines, with their respective receptors on the surface of these cells. ${ }^{11}$ In this context, Ceradini et $\mathrm{al}^{12}$ found that hypoxia-induced factor (HIF)- $1 \alpha$ increases the endothelial expression of stromal cell-derived factor (SDF)-1, resulting in homing of CXCR4-positive progenitor cells to areas of hypoxia.

Based on these findings, we hypothesized in the present study that EPCs may also be recruited into developing endometriotic lesions during vascularization. To test our hypothesis, we surgically induced i.p. endometriotic lesions in irradiated FVB/N mice that were reconstituted with bone marrow from FVB/N-TgN (Tie2/green fluorescent protein [GFP]) 287 Sato mice. These transgenic

Supported by the research program of the Medical Faculty of the University of Saarland, Homburg/Saar, Germany (HOMFOR 2008).

Accepted for publication September 21, 2010.

Address reprint requests to Matthias W. Laschke, M.D., Ph.D., Institute for Clinical and Experimental Surgery, University of Saarland, D-66421 Homburg/Saar, Germany. E-mail: matthias.laschke@uniklinik-saarland.de. 
mice express the reporter gene GFP under the transcriptional control of the Tie2 promoter. ${ }^{13}$ Tie2 is a vascular endothelial cell-specific receptor tyrosine kinase that controls numerous signaling pathways that are involved in endothelial cell migration, proliferation, and reorganization of the actin cytoskeleton. ${ }^{14}$ Accordingly, the presence of GFP signals in endometriotic lesions of wildtype mice that underwent transplantation with bone marrow from the transgenic strain allowed us to detect Tie2-positive bone marrow-derived EPCs. Because recruitment of EPCs into endometriotic lesions may be crucially dependent on the formation of a new microvasculature and establishment of blood perfusion during engraftment of ectopic endometrial tissue, we also analyzed the vascularization process of engrafting endometriotic lesions by immunohistochemistry and intravital fluorescence microscopy. ${ }^{15,16}$

\section{Materials and Methods}

\section{Animals}

This study was conducted in accordance with the German legislation on protection of animals and the $\mathrm{Na}$ tional Institutes of Health Guide for the Care and Use of Laboratory Animals (Institute of Laboratory Animal Resources, National Research Council, Washington, DC) and was approved by the local governmental animal care committee.

For the experiments, 12- to 16-week-old female C57BL/6 mice (Charles River, Sulzfeld, Germany), FVB/N mice, and FVB/N-TgN (Tie2/GFP) 287 Sato mice (Jackson Laboratories, Bar Harbor, ME), with a body weight of 20 to $25 \mathrm{~g}$, were used. They were maintained under controlled environmental conditions and had free access to tap water and standard pellet food (Altromin, Lage, Germany). To exclude discrepancies between individual animals because of different sex hormone levels, estrus cycling was evaluated by cytological analysis of vaginal lavage samples. For this purpose, $15 \mu \mathrm{L}$ of $0.9 \%$ saline was carefully pipetted into the vagina and subsequently transferred onto a glass slide for examination under a phase-contrast microscope (CH-2; Olympus, Hamburg, Germany). Only those animals that were in the stage of estrus were used for the experiments.

\section{Bone Marrow Transplantation}

Bone marrow cells were obtained by flushing the femurs and tibias of donor FVB/N-TgN (Tie2/GFP) 287 Sato mice with cold PBS. For generation of Tie2-GFP chimeras, wild-type FVB/N mice were lethally irradiated (total dose, 8.5 Gy). After 4 hours, the mice underwent transplantation with $2 \times 10^{7}$ bone marrow cells resuspended in 200 $\mu \mathrm{L}$ of cold PBS, via tail vein injection. Subsequently, the mice were left for 4 weeks to allow complete reconstitution of the bone marrow.
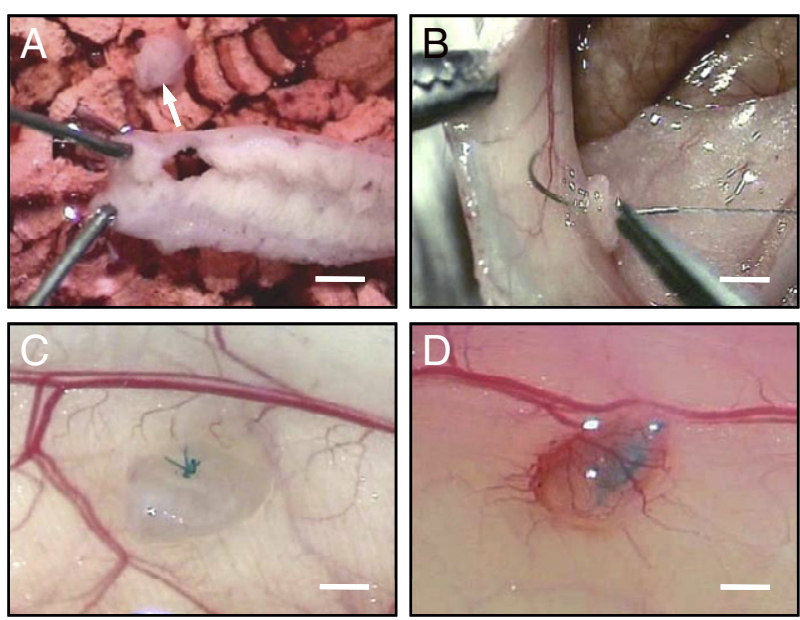

Figure 1. Surgical induction of i.p. endometriosis. A: For this purpose, uterine tissue samples of comparable size (arrow) are removed from longitudinally opened uterine horn of a donor mouse by a biopsy punch. B: The tissue samples are then sutured to the peritoneal wall of a recipient animal. $\mathbf{C}$ and $\mathbf{D}$ : Typical appearance of uterine tissue samples directly (C) and at day 28 (D) after fixation to the lateral abdominal wall. After 28 days, the tissue sample has developed to a well-vascularized endometriotic lesion (D). Scale bars: $2 \mathrm{~mm}(\mathbf{A}) ; 3.1 \mathrm{~mm}(\mathbf{B}) ; 1.3 \mathrm{~mm}(\mathbf{C}$ and $\mathbf{D})$.

\section{Model of i.p. Endometriosis}

Intraperitoneal endometriotic lesions were surgically induced by suturing uterine tissue samples to the abdominal wall. ${ }^{17}$ For syngeneic transplantation, FVB/N donor mice were anesthetized by i.p. injection of ketamine (75$\mathrm{mg} / \mathrm{kg}$ body weight; Pharmacia GmbH, Erlangen, Germany) and $2 \%$ xylazine (15-mg/kg body weight; Rompun, Bayer, Leverkusen, Germany). Both uterine horns were removed and transferred in a Petri dish containing $37^{\circ} \mathrm{C}$ warm Dulbecco's modified Eagle's medium (10\% fetal calf serum, 100-U/ml penicillin, and 0.1-mg/ml streptomycin; PAA, Cölbe, Germany). Subsequently, the uterine horns were opened longitudinally with microscissors under a stereomicroscope (M651; Leica Microsystems $\mathrm{GmbH}$, Wetzlar, Germany), and 2-mm tissue samples were removed using a dermal biopsy punch (Stiefel Laboratorium GmbH, Offenbach am Main, Germany) (Figure 1A). Then, four tissue samples were fixed with a 7-0 polypropylene suture (Prolene; Ethicon Products, Norderstedt, Germany) to the right and left abdominal wall of anesthetized Tie2-GFP bone marrow-transplanted animals through a midline incision (Figure 1, B-D). Finally, the laparotomy was closed with a 5-0 muscle and skin polypropylene suture (Prolene).

\section{Intravital Fluorescence Microscopy}

Vascularization of developing endometriotic lesions was analyzed by intravital fluorescence microscopy. For this purpose, the anesthetized animals were laparotomized and turned to their left or right side on a stage (Plexiglas) to position the endometriotic lesions under the microscope. To prevent desiccation of the lesions, the tissue was moistened with $37^{\circ} \mathrm{C}$ warm PBS during microscopy. After i.v. injection of $0.05-\mathrm{ml} 5 \%$ fluorescein isothiocyanate-labeled dextran 150,000 (contrast enhancement by 
intravascular staining of plasma), intravital fluorescence microscopy was performed (Zeiss Axiotech microscope; Zeiss, Oberkochen, Germany) with a 100-W mercury lamp attached to an epi-illumination filter block for blue, green, and UV light. The microscopic images were recorded by a charge-coupled device video camera (FK6990; Pieper, Schwerte, Germany) and transferred to a DVD system for off-line evaluation. By $\times 5, \times 10$, and $\times 20$ long-distance objectives (Zeiss), magnifications of $\times 115, \times 230$, and $\times 460$ were achieved on a 14-inch video screen (KV-14CT1E; Sony, Tokyo, Japan). At the end of microscopy, the animals were sacrificed with an overdose of pentobarbital, and the endometriotic lesions were carefully excised for further histological and immunohistochemical analyses.

Quantitative off-line analysis of the DVDs was performed by a computer-assisted image analysis system (Caplmage; Dr. Zeintl, Heidelberg, Germany) and included the determination of the fraction (percentage) of endometriotic lesions that exhibited red blood cell (RBC)-perfused microvessels, the functional capillary density (ie, the length of perfused microvessels per observation area $\left.\left[\mathrm{cm} / \mathrm{cm}^{2}\right]\right)$, the diameters of the microvessels $(\mu \mathrm{m})$, and the centerline RBC velocity $\left(V_{R B C}[\mu \mathrm{m} / \mathrm{s}]\right)$. In addition, volumetric blood flow of individual microvessels was calculated from $\mathrm{V}_{\mathrm{RBC}}$ and diameter $(d)$ for each microvessel as follows: Volumetric Blood Flow $=\pi \times(d / 2)^{2} \times V_{\mathrm{RBC}} / \mathrm{K}(\mathrm{pL} / \mathrm{s})$, where $K(1.3)$ represents the Baker/Wayland factor, ${ }^{18}$ considering the parabolic velocity profile of blood in microvessels. Wall shear rate $(y)$ was calculated based on the Newtonian definition: $y=8 \times \mathrm{V}_{\mathrm{RBC}} / d$.

\section{Histology and Immunohistochemistry}

For light microscopy, formalin-fixed specimens of endometriotic lesions were embedded in paraffin. Sections (thickness, $5 \mu \mathrm{m}$ ) were cut and stained with H\&E according to standard procedures. In addition, endometriotic lesions were embedded in embedding medium (Tissue TEK; R. Jung GmbH, Nussloch, Germany), snap frozen, and stored at $-80^{\circ} \mathrm{C}$. Subsequently, $5-\mu \mathrm{m}$-thick sections were cut on a cryostat (Leica, Nussloch, Germany) for immunohistochemical analyses. The GFP-positive cells within endometriotic lesions were detected using a rabbit polyclonal anti-GFP antibody as the primary antibody (1:200; Abcam, Cambridge, UK). A peroxidase-conjugated goat anti-rabbit antibody (1:200; Jackson, Suffolk, UK) served as the secondary antibody. To detect SDF1-expressing cells, a rabbit polyclonal anti-SDF-1 antibody (1:25, Abcam) was used as the primary antibody. A peroxidase-conjugated goat anti-rabbit antibody (1:100, Jackson) served as the secondary antibody. 3,3'-Diaminobenzidine tetrahydrochloride was used as chromogen. The sections were counterstained with $1 \%$ methyl green and examined by light microscopy (BX60; Olympus).

For immunofluorescence microscopy, cryosections were stained with a monoclonal rat anti-mouse antibody against CD31 (1:25; BD Pharmingen, Heidelberg, Germany) to detect endothelial cells within the tissue samples. A goat anti-rat Cyanine 3 (Сy3) GmbH, Hamburg,
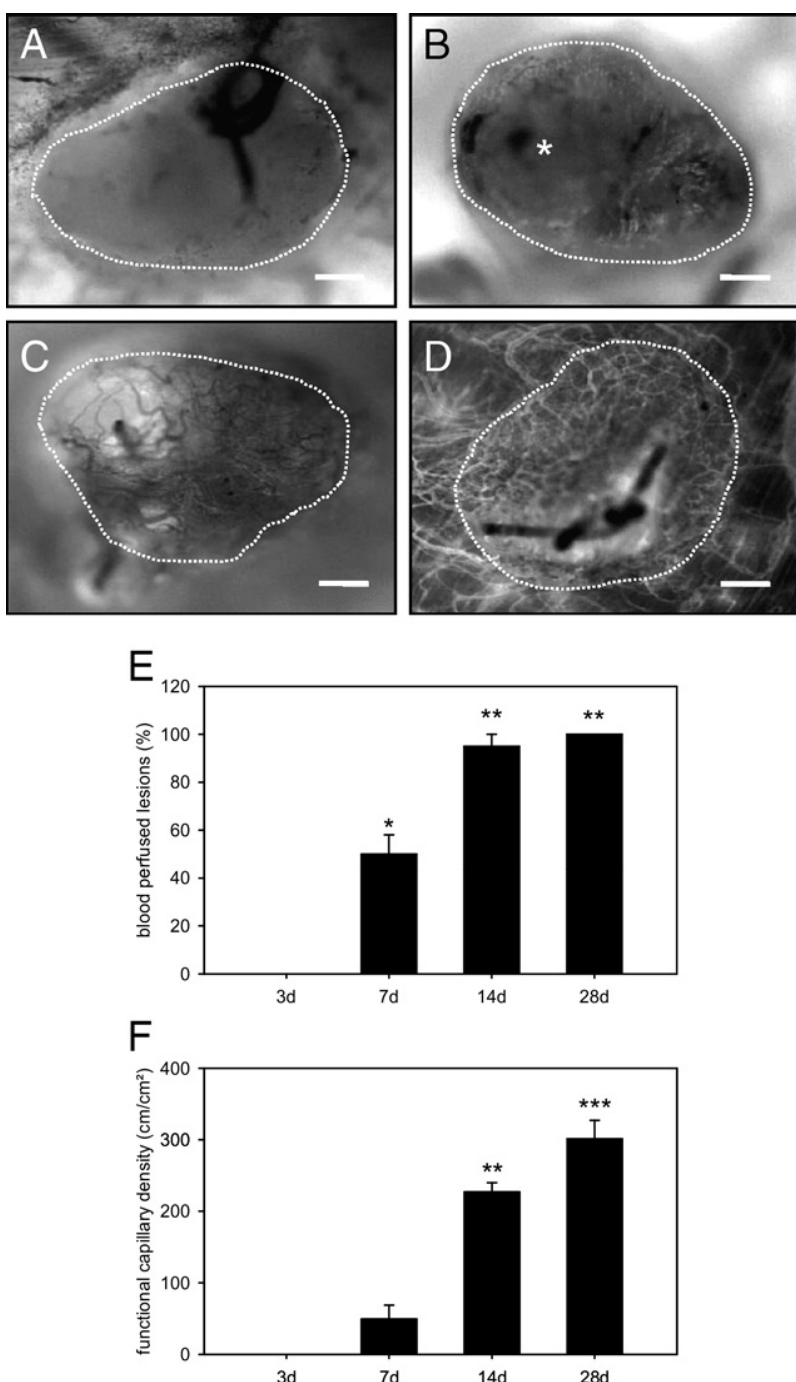

Figure 2. Intravital fluorescence microscopy of developing endometriotic lesions (borders marked by broken line) at days $3(\mathbf{A}), 7(\mathbf{B}), 14(\mathbf{C})$, and 28 (D) after fixation of uterine tissue samples from $\mathrm{FVB} / \mathrm{N}$ mice to the lateral abdominal wall of Tie2-GFP bone marrow-transplanted animals. Microvessels are lacking within the lesion at day 3 (A). After 7 days, numerous blood-perfused microvessels can be detected within the lesion, although a substantial part of the lesion still lacks vascularization (B asterisk). Endometriotic lesions at days 14 and 28 exhibit complete glomerulumlike microvasular networks ( $C$ and $\mathbf{D})$. Blue light epi-illumination with contrast enhancement by $5 \%$ fluorescein isothiocyanatelabeled dextran 150,000 i.v.. Scale bars $=250 \mu \mathrm{m}$. E and $\mathbf{F}$ : Fraction of blood-perfused lesions (\%) and functional capillary density $\left(\mathrm{cm} / \mathrm{cm}^{2}\right)$ of developing endometriotic lesions at days 3,7,14, and 28 after fixation of uterine tissue samples to the lateral abdominal wall, as assessed by intravital fluorescence microscopy and computer-assisted image analysis. Data are given as the mean \pm SEM. ${ }^{*} P<0.05$ versus day $3 ;{ }^{*} P<0.05$ versus days 3 and 7 ; ${ }^{* * *} P<0.05$ versus days 3,7 , and 14 .

Germany) served as the secondary antibody. Additional cryosections were stained with a monoclonal rat antimouse antibody against CD34 (1:30, Abcam) and a monoclonal rabbit anti-mouse antibody against vascular endothelial growth factor receptor (VEGFR)-2 (1:20, Abcam) for the detection of EPCs. As secondary antibodies, we used a goat anti-rat Cy3 antibody (1:600, dianova $\mathrm{GmbH}$ ) and a biotin-labeled goat anti-rabbit antibody (1:15, Jackson), which was detected by fluorescein-labeled streptavidin (1:50; Vector Labs, Burlingame, CA). Moreover, cryosections were stained with an antibody 
Table. Data on Microvessels for Developing Endometriotic Lesions at Days 7, 14, and 28 After Fixation of Uterine Tissue Samples

\begin{tabular}{lrrr}
\hline & \multicolumn{3}{c}{ Day } \\
\cline { 2 - 4 } Variable & \multicolumn{1}{c}{7} & 14 & 28 \\
\hline Microvessel diameter, $\mu \mathrm{m}$ & $23.9 \pm 1.1$ & $24.5 \pm 1.9$ & $26.9 \pm 1.6$ \\
RBC $_{\text {Rm } / \mathrm{s}}$ & $177.8 \pm 36.9$ & $344.2 \pm 97.6$ & $419.6 \pm 40.2$ \\
Volumetric blood flow, pL/s & $60.5 \pm 13.3$ & $119.6 \pm 18.8$ & $194.7 \pm 33.3^{\dagger}$ \\
Wall shear rate, $1 / \mathrm{s}$ & $60.9 \pm 12.4$ & $121.5 \pm 42.9$ & $127.7 \pm 13.8$ \\
\hline
\end{tabular}

Data are given as the mean $\pm \mathrm{SEM}$. Uterine tissue samples were taken from FVB/N mice and fixed to the lateral abdominal wall of Tie2-green fluorescent protein bone marrow-transplanted animals.

${ }^{\dagger} P<0.05$ versus days 7 and 14 .

$\mathrm{V}_{\mathrm{RBC}}$, red blood cell velocity.

against CD31, as previously described, and a monoclonal rabbit anti-mouse antibody against GFP (1:200, Abcam). As secondary antibody for GFP, a biotin-labeled goat anti-rabbit antibody (1:15, Jackson) was used, which was detected by fluorescein-labeled streptavidin (1:50, Vector Labs). On each section, cell nuclei were stained with Hoechst (1:500; Sigma, Deisenhofen, Germany) to merge the images exactly. The sections were examined using a microscope (BZ-8000; Keyence, Osaka, Japan).

Quantitative analyses of the sections included determination of the following: SDF-1-positive cells per lesion and fraction of GFP-positive cells of all endothelial cells lining the wall of microvessels within endometriotic lesions (percentages), GFP-positive cells per lesion \%o, and density of CD31-positive microvessels per lesion $\left(\mathrm{mm}^{-2}\right)$.

\section{Flow Cytometry}

To analyze whether endometriosis increases the number of EPCs in the peripheral blood and in hematopoietic organs of mice (ie, bone marrow and spleen), we performed flow cytometric analyses. For this purpose, endometriotic lesions were surgically induced in C57BL/6 mice, as previously described. Sham-operated on animals that underwent an identical laparotomy, however, with fixation of uterine tissue-free polypropylene sutures (Prolene) to the lateral abdominal wall, and a group of untreated animals served as controls. At days 3 and 14 after induction of endometriosis, blood, bone marrow, and spleen of the animals were harvested. After lysis of RBCs and Fc blockade (CD16/CD32, BD Pharmingen), the viable lymphocyte population was analyzed for the expression of the EPC markers Sca-1-fluorescein isothiocyanate (BD Pharmingen) and VEGFR-2-phycoerythrin (BD Pharmingen). Isotype-identical antibodies served as controls (rat IgG2a $a_{\kappa}$; BD Pharmingen). Two-color flow cytometric analyses were performed by a FACScan (BD Pharmingen). Data were evaluated by computer software (CellQuest; BD Pharmingen).

\section{Experimental Protocol}

A total of 80 endometriotic lesions were surgically induced in 20 Tie2-GFP bone marrow-transplanted animals. After 3, 7, 14, and 28 days, 20 endometriotic lesions of five animals at each point were analyzed by intravital fluorescence microscopy. In four lesions per point, the numbers of SDF-1-positive cells and GFP-positive cells and the density of CD31-positive microvessels were additionally assessed by immunohistochemistry.

In a second set of experiments, a total of 48 endometriotic lesions were surgically induced in 12 Tie2-GFP bone marrow-transplanted animals, which were treated daily with the SDF-1/CXCR4 axis antagonist AMD3100 $(n=6$; $5 \mathrm{mg} / \mathrm{kg}$ dissolved in 100- $\mu$ L PBS s.c.; Tocris Bioscience, Bristol, UK) or vehicle (control, $n=6$; $100-\mu$ L PBS s.c.). After 14 days, the functional capillary density and the number of GFP-positive endothelial cells of the endo-
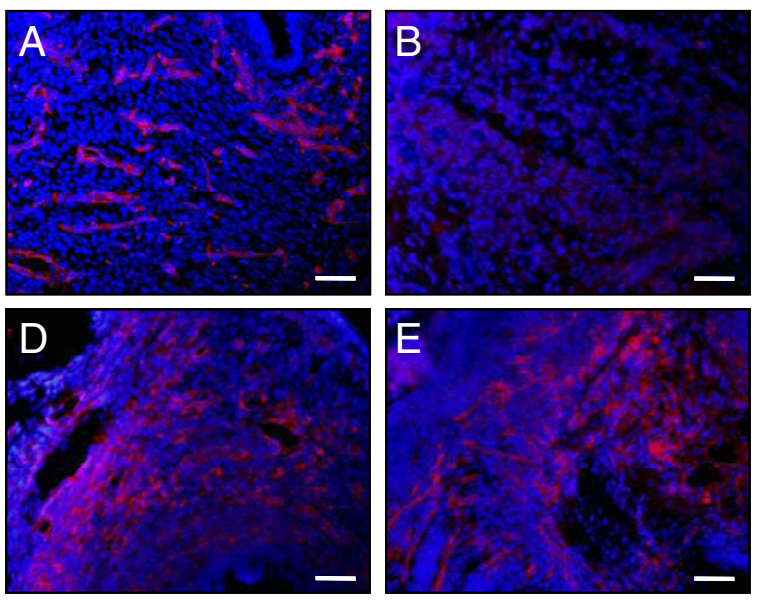
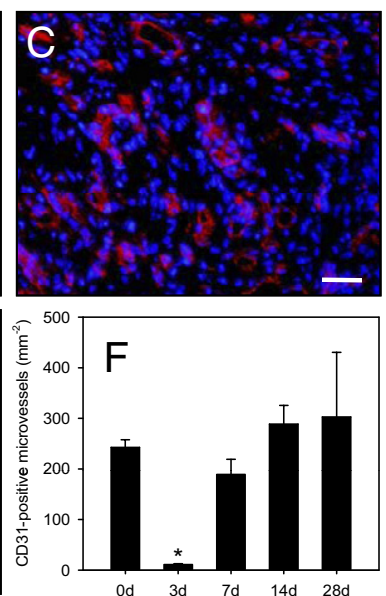

Figure 3. Immunohistochemical detection of microvessels in uterine tissue samples from $\mathrm{FVB} / \mathrm{N}$ mice directly before $(\mathbf{A})$ and at day $3(\mathbf{B})$ 7 (C), $14(\mathbf{D})$, and 28 (E) after transplantation into the peritoneal cavity of Tie2-GFP bone marrow-transplanted mice for the surgical induction of endometriotic lesions. Sections were stained with Hoechst to identify cell nuclei (blue) and an antibody against CD31 for the detection of endothelial cells (red). Scale bars $=50 \mu \mathrm{m}$. Density of CD31-positive microvessels $\left(\mathrm{mm}^{-2}\right)$ in uterine tissue samples directly before (day 0 ) and at day $3,7,14$, and 28 after transplantation $(\mathbf{F})$ Data are given as the mean \pm SEM. ${ }^{*} P<0.05$ versus days $0,7,14$, and 28 . 
metriotic lesions were assessed by intravital fluorescence microscopy and immunohistochemistry.

For flow cytometric analyses, 64 endometriotic lesions were surgically induced in $16 \mathrm{C} 57 \mathrm{BL} / 6$ mice. After 3 and 14 days, blood, bone marrow, and spleen of the animals were harvested to quantify the number of Sca-1/VEGFR-2-positive EPCs. Sham-operated on animals $(n=16)$ and untreated mice $(n=8)$ served as controls.

\section{Statistics}

Data were first analyzed for normal distribution and equal variance. Differences between two groups were assessed by the unpaired Student's t-test. Differences between multiple groups were calculated by analysis of variance, followed by the appropriate post hoc comparison (SigmaPlot 11.0; Systat Software, Chicago, IL). All data are given as mean \pm SEM. Statistical significance was accepted for $P<0.05$.

\section{Results}

\section{Vascularization of Endometriotic Lesions}

In the present study, we analyzed the development of microvascular networks and the establishment of blood flow in surgically induced i.p. endometriotic lesions using the technique of intravital fluorescence microscopy (Figure 2, A-D). We found that at day 3 after transplantation of uterine tissue samples into the peritoneal cavity, none of the developing endometriotic lesions exhibited blood-perfused microvessels; even after 7 days, only $50 \%$ of the lesions were already vascularized (Figure 2E). However, from day 14 on, almost all lesions presented with fully developed microvascular networks (Figure 2E). Throughout the observation period of 4 weeks, the functional capillary density of these networks progressively increased up to $300 \mathrm{~cm} / \mathrm{cm}^{2}$ (Figure 2F).

Microvessels of endometriotic lesions exhibited diameters of approximately 24 to $27 \mu \mathrm{m}$, without significant differences over time (Table). Within these microvessels, $V_{\mathrm{RBC}}$ increased from approximately $178 \mu \mathrm{m} / \mathrm{s}$ at day 7 to approximately $420 \mu \mathrm{m} / \mathrm{s}$ at day 28 (Table). Accordingly, calculated values of volumetric blood flow and wall shear rate were markedly elevated at day 28 when compared with day 7 (Table).

Additional immunohistochemical analyses further revealed that freshly isolated uterine tissue samples exhibited a dense vascularization of approximately 240 CD31positive microvessels per square millimeter (Figure 3, A and F). However, at day 3 after transplantation of the samples into the peritoneal cavity, almost all of the microvessels were no longer detectable, indicating a rapid loss of tissue-resident CD31-expressing endothelial cells during the initial phase of engraftment (Figure 3, B and F). Throughout the further course of the experiment, the number of CD31-positive microvessels within the developing endometriotic lesions progressively increased because of the ingrowth of newly formed blood vessels from

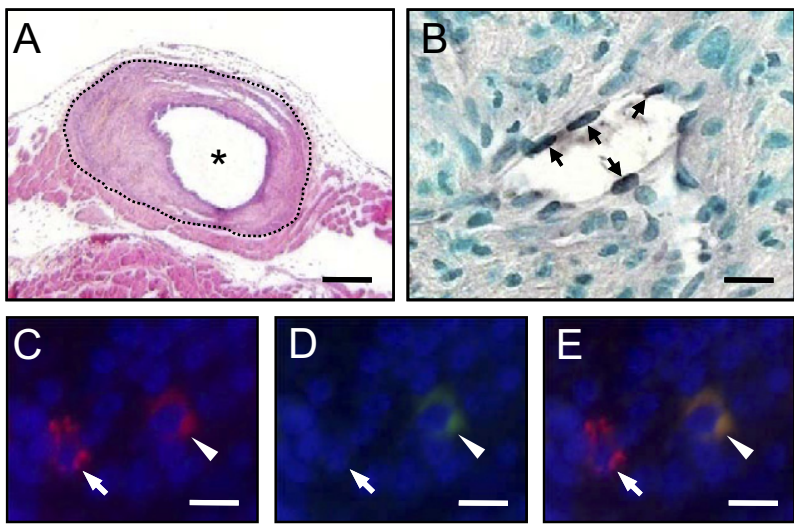

$\mathrm{F}$

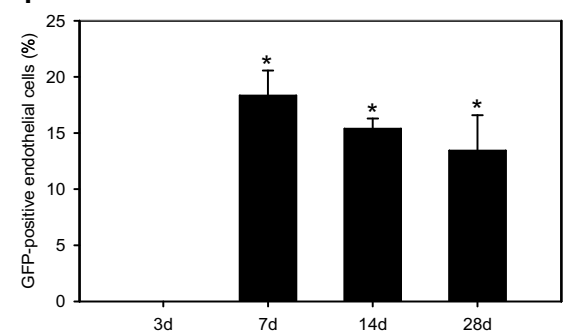

Figure 4. A: Hematoxylin-eosin-stained cross section of an i.p. endometriotic lesion (borders marked by broken line) at day 28 after fixation of an uterine tissue sample from an $\mathrm{FVB} / \mathrm{N}$ mouse to the lateral abdominal wall of a Tie2-GFP bone marrow-transplanted animal. The lesion is characterized by a cystlike dilated endometrial gland (asterisk), which is surrounded by a well-vascularized endometrial stroma. B: Immunohistochemical detection of GFP-positive endothelial cells (arrows) of a blood vessel within an endometriotic lesion at day 28 , representing recruited bone marrow-derived EPCs. C through E: Immunofluorescence microscopy of an endometriotic lesion at day 14 after transplantation of an uterine tissue sample from an $\mathrm{FVB} / \mathrm{N}$ mouse into the peritoneal cavity of a Tie2-GFP bone marrow-transplanted animal. Histological sections were stained with Hoechst to identify cell nuclei (C-E, blue), an antibody against the endothelial cell marker CD31 $(\mathbf{C}, \mathbf{E}$, red) and an antibody against GFP (D, E, green). E displays the merge of $\mathbf{C}$ and $\mathbf{D}$. The EPC, which stains double positive for CD31/GFP (arrowheads), is next to a normal CD31-positive endothelial cell (arrows). Scale bars: $200 \mu \mathrm{m}$ (A); $8 \mu \mathrm{m}$ (B); $7 \mu \mathrm{m}(\mathbf{C}-\mathbf{E})$. F: Number (percentage) of GFP-positive endothelial cells lining the wall of blood vessels in endometriotic lesions at days $3,7,14$, and 28 after fixation of uterine tissue samples from $\mathrm{FVB} / \mathrm{N}$ mice to the lateral abdominal wall of Tie2-GFP bone marrowtransplanted animals. Data are given as the mean \pm SEM. ${ }^{*} P<0.05$ versus day 3 .

the surrounding host tissue, resulting in a final density of approximately 300 CD31-positive microvessels per square millimeter at day 28 (Figure 3, C-F).

\section{Recruitment of EPCs into Endometriotic Lesions}

Histological examination results of uterine tissue samples at day 28 after transplantation into the peritoneal cavity revealed that they had developed to endometriotic lesions of typical histological appearance (ie, cystlike dilated endometrial glands surrounded by a well-vascularized endometrial stroma; Figure 4A). At day 3 , we did not find any GFP-positive cells within these developing lesions. However, between days 7 and 28 , we could detect approximately $1 \%$ o GFP-positive cells without marked differences between the lesions at individual points. More detailed analysis of the localization of these cells revealed that approximately 95\% of them were incorporated into the endothelial lining of the microvessels (Fig- 

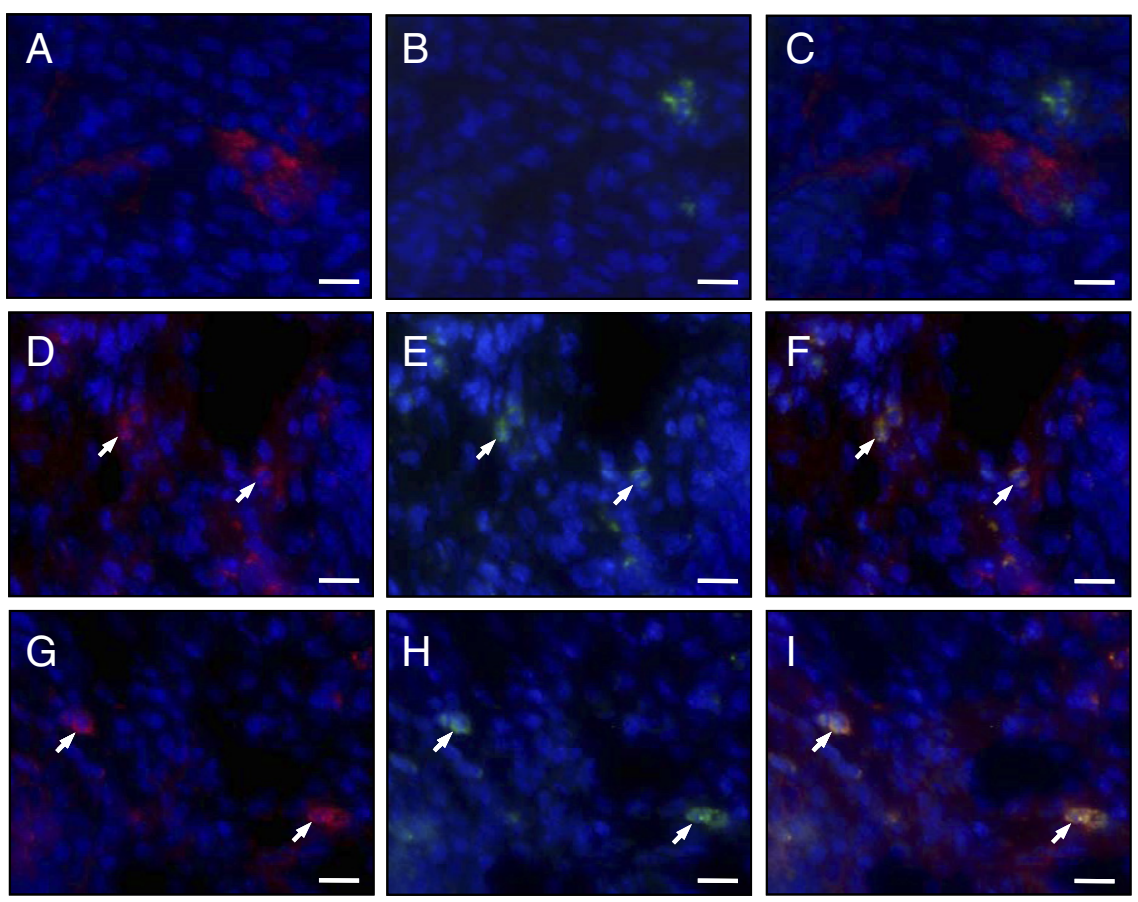

Figure 5. Immunohistochemical detection of CD34 and VEGFR-2-expressing cells in uterine tissue samples from $\mathrm{FVB} / \mathrm{N}$ mice directly before (A-C) and at days 7 (D-F) and $28(\mathbf{G}-\mathbf{I})$ after transplantation into the peritoneal cavity of Tie2GFP bone marrow-transplanted mice for the surgical induction of endometriotic lesions. Histological sections were stained with Hoechst to identify cell nuclei (A-I, blue), an antibody against CD34 (A, C, D, F, G, I, red), and an antibody against VEGFR-2 $(\mathbf{B}, \mathbf{C}, \mathbf{E}, \mathbf{F}, \mathbf{H}, \mathbf{I}$, green). $\mathbf{C}, \mathbf{F}$, and $\mathbf{I}$ display merges of the following: $\mathbf{A}$ and $\mathbf{B}, \mathbf{D}$ and $\mathbf{E}$, and $\mathbf{G}$ and $\mathbf{H}$, respectively. The freshly isolated uterine tissue sample only contains cells that are either positive for CD34 or VEGFR-2 (A-C), whereas the uterine tissue grafts from days 7 and 28 contain several CD34/VEGFR-2-positive EPCs (D-I, arrows). Scale bars $=20 \mu \mathrm{m}$. ure 4B), whereas approximately $5 \%$ were sporadically distributed in the surrounding endometrial stroma. Accordingly, we also analyzed the fraction of GFP-positive cells lining the vessel walls. Immunohistochemical analyses revealed that these cells stained double positive for GFP and CD31, indicating that they represented bone marrow-derived EPCs, which contributed to the microvasculature of developing endometriotic lesions (Figure 4, C-E). Interestingly, $18 \%$ of all endothelial cells were GFP positive at day 7 after the induction of endometriotic lesions (Figure 4F). Throughout the further time course, this cell fraction slightly decreased to $13 \%$ at day 28 .

In addition to circulating bone marrow-derived EPCs, local tissue-resident EPCs may have contributed to the vascularization of endometriotic lesions in our endometriosis model of uterine tissue transplantation. To clarify this issue, we analyzed the distribution of CD34/VEGFR2-positive EPCs in uterine tissue samples before and after transplantation into the peritoneal cavity. Interestingly, we found that freshly isolated uterine tissue samples and samples that were transplanted for 3 days into the peritoneal cavity did not contain any CD34/NEGFR2-positive cells (Figure 5, A-C). In contrast, we could detect CD34/VEGFR-2-positive EPCs in developing endometriotic lesions at day 7 to 28 after transplantation of uterine tissue samples into the peritoneal cavity (Figure 5, D-I), which correlated well with our results regarding the homing of circulating bone marrow-derived EPCs into the lesions.

\section{SDF-1 Expression in Endometriotic Lesions}

The chemokine SDF-1 has previously been described as an important chemotactic factor for homing of EPCs to sites of ischemia. $^{12}$ Therefore, in the present study, we analyzed
SDF-1 expression in developing endometriotic lesions. At day 3 after transplantation of uterine tissue samples into the peritoneal cavity, only $5 \%$ of the cells were positively stained for SDF-1 (Figure 6, A and E). In contrast, we could detect SDF-1 expression in almost $75 \%$ of the cells at day 7 (Figure $6, B$ and $E$ ). Throughout the end of the observation period of 4 weeks, this high SDF-1 expression decreased again to $4 \%$ at day 28 (Figure 6, C-E).

\section{Recruitment of EPCs and Lesion Vascularization in AMD3100-Treated Animals}

To analyze the contribution of circulating bone marrowderived EPCs to the vascularization of endometriotic lesions, we compared mice that were treated with the SDF1/CXCR4 axis antagonist AMD3100 with vehicle-treated control animals. We found that treatment with AMD3100 significantly reduced the number of GFP-positive endothelial cells in developing endometriotic lesions at day 14 after transplantation of uterine tissue samples into the peritoneal cavity when compared with controls (Figure 7A). Intravital fluorescence microscopic analysis of the lesions further showed that this was associated with a significantly decreased functional capillary density of the lesions (Figure 7B). Taken together, these results indicate that EPCs are essential for the vascularization of endometriotic lesions.

\section{EPC Levels in Peripheral Blood and Hematopoietic Organs of Endometriotic and Control Mice}

In an additional set of experiments, we analyzed by flow cytometry whether the engraftment of endometriotic le- 

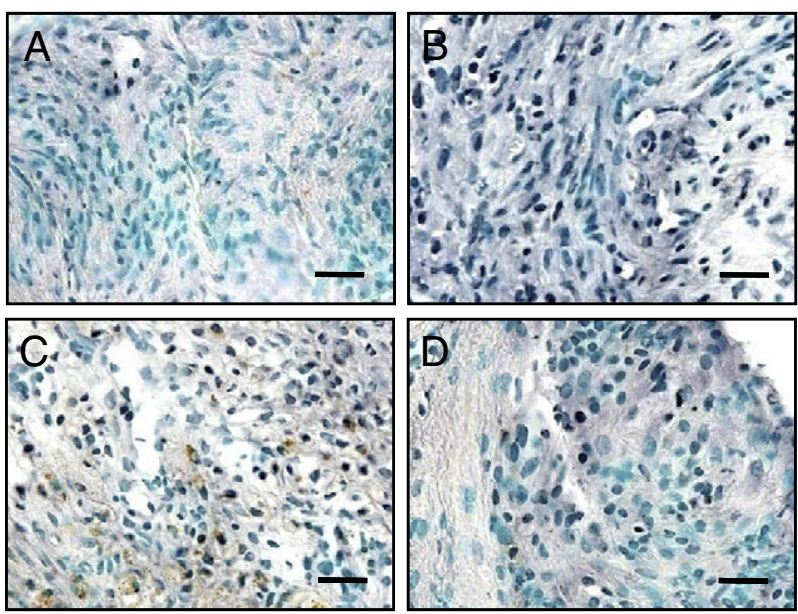

E

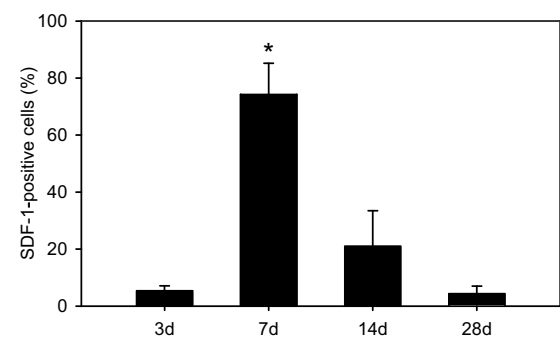

Figure 6. Immunohistochemical detection of SDF-1-expressing cells in endometriotic lesions at days $3(\mathbf{A}), 7(\mathbf{B}), 14(\mathbf{C})$, and 28 (D) after fixation of uterine tissue samples from $\mathrm{FVB} / \mathrm{N}$ mice to the lateral abdominal wall of Tie2-GFP bone marrow-transplanted animals. Scale bars $=30 \mu \mathrm{m}$. Number (percentage) of SDF-1-positive cells in endometriotic lesions at days 3, 7, 14, and $28(\mathbf{E})$. Data are given as the mean \pm SEM. ${ }^{*} P<0.05$ versus days 3,14 , and 28 .

sions is associated with increased levels of EPCs in the blood, bone marrow, and spleen of C57BL/6 mice. Based on our intravital microscopic findings, we studied an early point (ie, day 3) and a point at which endometriotic lesions had already established their own blood supply (ie, day 14) after transplantation of uterine tissue samples. We found that the induction of endometriotic lesions did not increase the number of circulating Sca-1/NEGFR-2positive EPCs in the peripheral blood at both observation points (Figure 8A). In the bone marrow, the number of EPCs in endometriotic mice was significantly increased at day 3 when compared with day 14 (Figure 8B). However, because comparable results were also found for sham-operated animals, the EPC increase in the bone marrow has to be interpreted as a response to the surgical trauma and not to the induction of endometriotic lesions. In addition, EPC levels in the spleen of endometriotic, sham-operated, and control animals did not show any significant differences at both observation points (Figure 8C).

\section{Discussion}

In the present study, we provide the proof of principle that bone marrow-derived circulating EPCs are recruited and incorporated into the vasculature of endometriotic lesions. Therefore, our work indicates, for the first time to our knowl- edge, that in addition to angiogenesis (ie, the development of new blood vessels from preexisting ones), the process of vasculogenesis (ie, de novo generation of endothelial cells from EPCs) may represent an integral mechanism in the pathogenesis of endometriosis.

During the past decade, numerous studies have shown that EPCs contribute to the vascularization of tumors and ischemic tissues. ${ }^{5}$ However, the results about the extent to which circulating EPCs are incorporated into the endothelium of newly forming blood vessels are controversial. A few studies ${ }^{19,20}$ reported that $50 \%$ to $100 \%$ of the tumor vasculature may originate from EPCs, whereas most studies ${ }^{21}$ indicated that only rare numbers of EPCs can be detected within the developing microvascular networks of growing tumors. In the present study, we found that approximately $15 \%$ of all endothelial cells within engrafting endometriotic lesions are derived from circulating EPCs. This is a relatively high fraction, considering the fact that irradiation required for bone marrow transplantation likely had a significant effect on the production of proangiogenic growth factors and chemotactic mediators of EPC recruitment, even underestimating the number of EPCs incorporated into the vasculature of endometriotic lesions in nonirradiated animals. However, endometriotic lesions originate from shed endometrium, which is a highly regenerative tissue type and a rich source of mesenchymal stem cells. ${ }^{22,23}$ Moreover, it has already been shown to be the target tissue for the engraftment of bone marrow-derived stem cells. ${ }^{24}$ Thus, it is possible that endometrial tissue is well adapted to the process of stem cell homing. Moreover, our results are in line with other studies that focused on the recruitment of bone marrow-derived stem cells into the vasculature of transplanted ischemic tissues. For instance, Capla et al ${ }^{25}$ reported that EPCs contribute to $15 \%$ to $20 \%$ of endothelial cells in blood vessels of freely transplanted skin grafts.

In our experiments, we induced endometriotic lesions in Tie2-GFP bone marrow-transplanted animals. Notably,

A

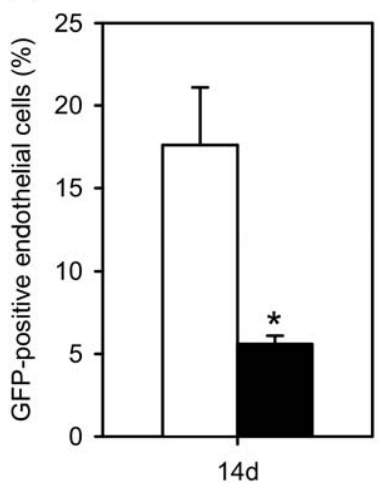

$B$

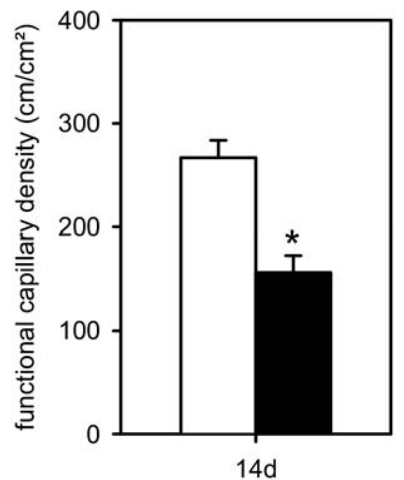

Figure 7. A and B: Number (percentage) of GFP-positive endothelial cells lining the wall of blood vessels and functional capillary density $\left(\mathrm{cm} / \mathrm{cm}^{2}\right)$ of developing endometriotic lesions at day 14 after fixation of uterine tissue samples from FVB/N mice to the lateral abdominal wall of Tie2-GFP bone marrow-transplanted animals that were treated daily with the SDF-1/CXCR4 axis antagonist AMD3100 (black bars) or vehicle (white bars), as assessed by immunohistochemistry (A) or intravital fluorescence microscopy and computer-assisted image analysis $(\mathbf{B})$. Data are given as the mean \pm SEM ${ }^{*} P<0.05$ versus the vehicle-treated control. 

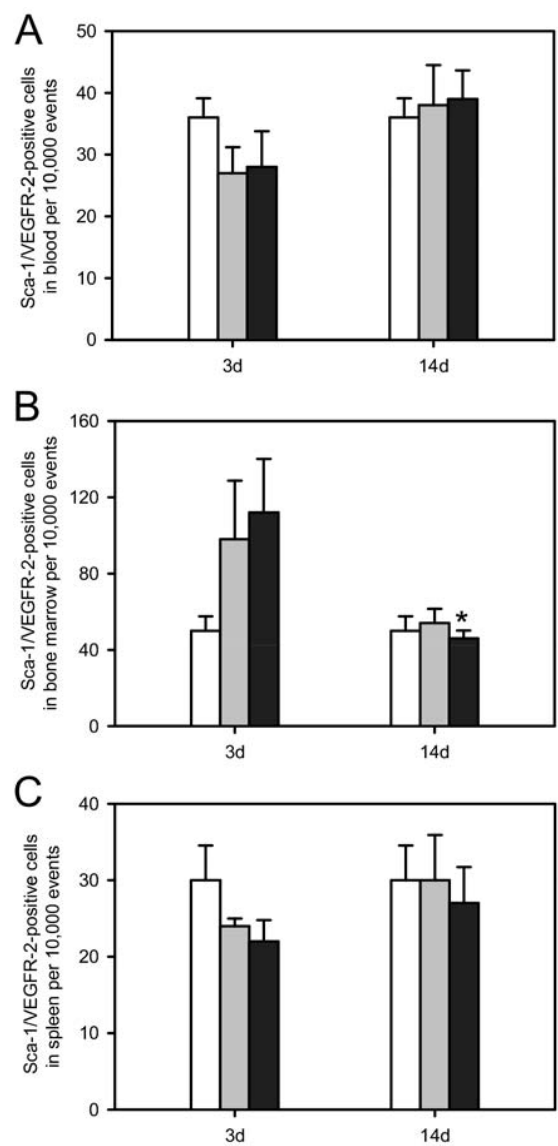

Figure 8. Number of Sca-1/VEGFR-2-positive EPCs per 10,000 events in blood (A), bone marrow (B), and spleen $(\mathbf{C})$ of endometriotic (black bars), sham-operated (gray bars), and untreated (white bars) C57BL/6 mice at days 3 and 14 , as assessed by flow cytometry. Data are given as the mean \pm SEM. ${ }^{*} P<0.05$ versus day 3 .

we found that almost all GFP-positive cells inside the lesions were incorporated into the microvascular endothelium. However, a small fraction of GFP-positive cells was located within the endometrial stroma surrounding the blood vessels. Therefore, these cells may not have belonged to the pool of circulating EPCs. This interpretation is not unlikely because studies with the FVB/N-TgN (Tie2/GFP) 287 Sato mouse have revealed a subset of Tie2-GFP-positive cells in the bone marrow; these cells coexpress the hematopoietic marker CD45 and the endothelial markers CD34, VEGFR2, and CD31. ${ }^{26}$ Accordingly, there may be a common hemangioblastic precursor cell that produces both EPCs and hematopoietic stem cells that subsequently differentiate into lymphoid and myeloid cells. Alternatively, there may be separate angioblastic and hematopoietic precursors. The Tie2-GFPpositive cell population of the Sato mouse is clearly heterogeneous, which may explain our observation that not all circulating GFP-positive cells were incorporated as EPCs into the microvascular endothelium of endometriotic lesions.

Recruitment of EPCs into ischemic tissues is critically dependent on blood perfusion and associated remodeling processes in the microvasculature. ${ }^{25}$ Therefore, in the present study, we analyzed the vascularization of endo- metriotic lesions using intravital fluorescence microscopy and immunohistochemistry. In doing so, we could demonstrate that in our endometriosis model the microvasculature of transplanted uterine tissue samples underwent drastic changes, including the loss of tissue-resident CD31-positive microvessels and the establishment of a completely new microvasculature with ingrowth of blood vessels from the surrounding host tissue. Moreover, we found that recruitment of circulating EPCs in developing endometriotic lesions clearly coincided with the establishment of own blood supply at day 7 after free transplantation of the tissue samples into the peritoneal cavity. In fact, before this observation point, we did not detect any CD34/VEGFR-2-positive EPCs in the grafts, demonstrating that neither circulating bone marrowderived EPCs nor local tissue-resident EPCs contributed to the initiation of lesion vascularization by paracrine effects before the establishment of microvascular blood perfusion.

We also detected the highest levels of SDF-1 expression inside the lesions at day 7, indicating that the necessary signals for the trafficking of EPCs to the ectopic endometrial tissue may require reestablishment of some circulation. We could further show that treatment with the SDF-1/CXCR4 axis antagonist AMD3100 significantly decreased the recruitment of circulating EPCs. More important, this was associated with significantly decreased functional capillary density of the uterine tissue grafts, which underlines the essential contribution of bone marrow-derived EPCs to the establishment of a dense microvasculature of developing endometriotic lesions.

Because the fraction of GFP-positive endothelial cells remained nearly constant between days 14 and 28 despite decreasing SDF-1 levels and an increasing functional capillary density in the developing endometriotic lesions, we suggest that, in addition to hypoxia-induced SDF-1 signaling, other mechanisms must have contributed to the recruitment of EPCs. These mechanisms may include shear stress-induced up-regulation of adhesion molecules of the selectin and integrin family, which have been shown to mediate EPC arrest to the endothelium, ${ }^{27,28}$ or capture of EPCs from the bloodstream by activated platelets, which adhere to the wall of ischemically injured microvessels. ${ }^{29}$

Circulating EPCs have been markedly increased in the bloodstream after myocardial infarction, bypass surgery, or physical training. ${ }^{30,31}$ Accordingly, we further analyzed in our study whether endometriosis is also associated with increased circulating EPCs, which may be used as a diagnostic biomarker for the disease. However, endometriotic and control mice did not show any differences. Moreover, we did not detect an increased number of EPCs in the bone marrow and spleen of endometriotic mice. These findings indicate that the physiological pool of circulating EPCs seems to be sufficient for significant accumulation and recruitment of EPCs in the microvasculature of developing endometriotic lesions. Nevertheless, we analyzed EPC numbers only at two points during the early phase of disease induction. Therefore, we cannot exclude that, under clinical conditions, EPC numbers 
are elevated in patients with chronic disease or severe stages of endometriosis.

In summary, in the present study, we demonstrated, for the first time to our knowledge, that EPCs contribute to the vascularization of endometriotic lesions. This finding highlights the process of vasculogenesis as an integral part of the pathogenesis of endometriosis and may also open the doors for the establishment of new diagnostic and therapeutic strategies for this frequent gynecological disease.

\section{Acknowledgments}

We are grateful for the technical assistance of Janine Becker, Julia Parakenings, and Sandra Schuler, Institute for Clinical and Experimental Surgery, Homburg/Saar, Germany.

\section{References}

1. Sampson JA: Peritoneal endometriosis due to menstrual dissemination of endometrial tissues into the peritoneal cavity. Am J Obstet Gynecol 1927, 14:422-469

2. Groothuis PG, Nap AW, Winterhager E, Grümmer R: Vascular development in endometriosis. Angiogenesis 2005, 8:147-156

3. Laschke MW, Menger MD: In vitro and in vivo approaches to study angiogenesis in the pathophysiology and therapy of endometriosis. Hum Reprod Update 2007, 13:331-342

4. Asahara T, Murohara T, Sullivan A, Silver M, van der Zee R, Li T, Witzenbichler B, Schatteman G, Isner JM: Isolation of putative progenitor endothelial cells for angiogenesis. Science 1997, 275:964967

5. Kopp HG, Ramos CA, Rafii S: Contribution of endothelial progenitors and proangiogenic hematopoietic cells to vascularization of tumor and ischemic tissue. Curr Opin Hematol 2006, 13:175-181

6. Sata M, Saiura A, Kunisato A, Tojo A, Okada S, Tokuhisa T, Hirai H, Makuuchi M, Hirata Y, Nagai R: Hematopoietic stem cells differentiate into vascular cells that participate in the pathogenesis of atherosclerosis. Nat Med 2002, 8:403-409

7. Kocher AA, Schuster MD, Szabolcs MJ, Takuma S, Burkhoff D, Wang J, Homma S, Edwards NM, Itescu S: Neovascularization of ischemic myocardium by human bone-marrow-derived angioblasts prevents cardiomyocyte apoptosis, reduces remodeling and improves cardiac function. Nat Med 2001, 7:430-436

8. Matsumoto T, Kuroda R, Mifune Y, Kawamoto A, Shoji T, Miwa M, Asahara T, Kurosaka M: Circulating endothelial/skeletal progenitor cells for bone regeneration and healing. Bone 2008, 43:434-439

9. Grant MB, May WS, Caballero S, Brown GA, Guthrie SM, Mames RN, Byrne BJ, Vaught T, Spoerri PE, Peck AB, Scott EW: Adult hematopoietic stem cells provide functional hemangioblast activity during retinal neovascularization. Nat Med 2002, 8:607-612

10. Peters BA, Diaz LA, Polyak K, Meszler L, Romans K, Guinan EC, Antin $\mathrm{JH}$, Myerson D, Hamilton SR, Vogelstein B, Kinzler KW, Lengauer C: Contribution of bone marrow-derived endothelial cells to human tumor vasculature. Nat Med 2005, 11:261-262

11. Hristov $M$, Weber $\mathrm{C}$ : Progenitor cell trafficking in the vascular wall. J Thromb Haemost 2009, 7(Suppl 1):31-34

12. Ceradini DJ, Kulkarni AR, Callaghan MJ, Tepper OM, Bastidas N, Kleinman ME, Capla JM, Galiano RD, Levine JP, Gurtner GC: Progenitor cell trafficking is regulated by hypoxic gradients through HIF-1 induction of SDF-1. Nat Med 2004, 10:858-864
13. Motoike T, Loughna S, Perens E, Roman BL, Liao W, Chau TC Richardson CD, Kawate T, Kuno J, Weinstein BM, Stainier DY, Sato TN: Universal GFP reporter for the study of vascular development. Genesis 2000, 28:75-81

14. Loughna S, Sato TN: Angiopoietin and Tie signaling pathways in vascular development. Matrix Biol 2001, 20:319-325

15. Laschke MW, Elitzsch A, Vollmar B, Menger MD: In vivo analysis of angiogenesis in endometriosis-like lesions by intravital fluorescence microscopy. Fertil Steril 2005, 84(Suppl 2):1199-1209

16. Laschke MW, Schwender C, Scheuer C, Vollmar B, Menger MD: Epigallocatechin-3-gallate inhibits estrogen-induced activation of endometrial cells in vitro and causes regression of endometriotic lesions in vivo. Hum Reprod 2008, 23:2308-2318

17. Laschke MW, Körbel C, Rudzitis-Auth J, Gashaw I, Reinhardt M, Hauff P, Zollner TM, Menger MD: High-resolution ultrasound imaging: a novel technique for the noninvasive in vivo analysis of endometriotic lesion and cyst formation in small animal models. Am J Pathol 2010 176:585-593

18. Baker M, Wayland $\mathrm{H}$ : On-line volume flow rate and velocity profile measurement for blood in microvessels. Microvasc Res 1974, 7:131-143

19. Lyden D, Hattori K, Dias S, Costa C, Blaikie P, Butros L, Chadburn A, Heissig B, Marks W, Witte L, Wu Y, Hicklin D, Zhu Z, Hackett NR, Crystal RG, Moore MA, Hajjar KA, Manova K, Benezra R, Rafii S: Impaired recruitment of bone-marrow-derived endothelial and hematopoietic precursor cells blocks tumor angiogenesis and growth. Nat Med 2001, 7:1194-1201

20. Garcia-Barros M, Paris F, Cordon-Cardo C, Lyden D, Rafii S, HaimovitzFriedman A, Fuks Z, Kolesnick R: Tumor response to radiotherapy regulated by endothelial cell apoptosis. Science 2003, 300:1155-1159

21. Ahn GO, Brown JM: Role of endothelial progenitors and other bone marrow-derived cells in the development of the tumor vasculature. Angiogenesis 2009, 12:159-164

22. Gargett CE: Uterine stem cells: what is the evidence? Hum Reprod Update 2007, 13:87-101

23. Gargett CE, Schwab KE, Zillwood RM, Nguyen HP, Wu D: Isolation and culture of epithelial progenitors and mesenchymal stem cells from human endometrium. Biol Reprod 2009, 80:1136-1145

24. Du H, Taylor HS: Contribution of bone marrow-derived stem cells to endometrium and endometriosis. Stem Cells 2007, 25:2082-2086

25. Capla JM, Ceradini DJ, Tepper OM, Callaghan MJ, Bhatt KA, Galiano RD, Levine JP, Gurtner GC: Skin graft vascularization involves precisely regulated regression and replacement of endothelial cells through both angiogenesis and vasculogenesis. Plast Reconstr Surg 2006, 117:836-844

26. Shaw JP, Basch R, Shamamian P: Hematopoietic stem cells and endothelial cell precursors express Tie-2, CD31 and CD45. Blood Cells Mol Dis 2004, 32:168-175

27. Hristov M, Zernecke A, Liehn EA, Weber C: Regulation of endothelial progenitor cell homing after arterial injury. Thromb Haemost 2007 98:274-277

28. Zampetaki A, Kirton JP, Xu Q: Vascular repair by endothelial progenitor cells. Cardiovasc Res 2008, 78:413-421

29. Lev El, Estrov Z, Aboulfatova K, Harris D, Granada JF, Alviar C Kleiman NS, Dong JF: Potential role of activated platelets in homing of human endothelial progenitor cells to subendothelial matrix. Thromb Haemost 2006, 96:498-504

30. Shintani S, Murohara T, Ikeda H, Ueno T, Honma T, Katoh A, Sasaki K, Shimada T, Oike Y, Imaizumi T: Mobilization of endothelial progenitor cells in patients with acute myocardial infarction. Circulation 2001 103:2776-2779

31. Laufs U, Werner N, Link A, Endres M, Wassmann S, Jürgens K, Miche E, Böhm M, Nickenig G: Physical training increases endothelial progenitor cells, inhibits neointima formation, and enhances angiogenesis. Circulation 2004, 109:220-226 